\title{
Labyrinthe
}

29 | 2008 (1)

Ce que le libéralisme promet

\section{L'histoire du droit comme histoire des contraintes discursives}

Sur le livre de Michel Troper, Terminer la Révolution. La Constitution de 1795

Diogo Sardinha

\section{CpenEdition}

Journals

Édition électronique

URL : http://journals.openedition.org/labyrinthe/3513

DOI : $10.4000 /$ labyrinthe.3513

ISSN : 1950-6031

Éditeur

Hermann

Édition imprimée

Date de publication : 11 janvier 2008

Pagination : 139-146

ISBN : 9782952613163

Référence électronique

Diogo Sardinha, "L'histoire du droit comme histoire des contraintes discursives », Labyrinthe [En ligne], 29 | 2008 (1), mis en ligne le 11 janvier 2008, consulté le 18 novembre 2020. URL : http:// journals.openedition.org/labyrinthe/3513; DOI : https://doi.org/10.4000/labyrinthe.3513 


\section{L'HISTOIRE DU DROIT COMME HISTOIRE DES CONTRAINTES DISCURSIVES Sur le livre de Michel Troper, Terminer la Révolution. La Constitution de 1795 .}

Pour donner un titre à son livre, Michel Troper - l'un des historiens et théoriciens du droit les plus respectés en France - s'est inspiré d'un autre titre, celui qu'a donné Pierre-Charles-Louis Baudin au texte présenté à la Convention le 18 août 1795 : «Rapport sur les moyens de terminer la révolution». La désignation impressionne, tant elle proclame sans ambages le but des Thermidoriens, que le même Baudin résume alors par les mots suivants : «Il est temps que la réalité succède aux passions» et que s'arrête «l'anarchie révolutionnaire affaissée sous le poids de ses propres excès » (p. 624). Nous sommes encore en pleine effervescence politique et sociale quand ce discours est prononcé. C'est pourquoi le simple fait qu'un représentant de ceux qui ont vaincu la Terreur puisse tenir de tels propos semble indiquer qu'une page est tournée pour de bon. Pour le dire avec plus de rigueur, le discours de Baudin qui justifie la nouvelle Constitution approuvée en 1975 (l'an III) peut porter à croire, avec d'autres éléments historiques, que ce texte fondamental représente une rupture profonde non seulement avec le gouvernement de type révolutionnaire, mais encore avec les termes de la Constitution montagnarde - démocratique et universaliste - de 1793.

Pourtant, les choses ne sont pas aussi simples. C'est en tout cas ce que prétend l'auteur de ce livre, qui s'engage alors dans une analyse détaillée des documents de l'époque afin de démontrer son hypothèse,

* Paris, Fayard, coll. «Histoire des Constitutions de la France», 2006, 779 p., $36 €$. 
selon laquelle la Constitution de 1795 représente certes «une rupture dans le contenu » avec la Constitution précédente, mais une rupture qui est assortie d'une «continuité dans les principes» (p. 16). Plus précisément, «sans être radicalement nouvelle, [la Constitution de 1795] n'est plus tout à fait la même et constitue elle-même une étape importante pour les développements constitutionnels futurs» (p. 90). Le problème se pose alors de savoir dans quelle mesure exacte elle se distingue de celle qui l'a précédée et, ce faisant, de mieux cerner le statut du texte de 1795. D'emblée nous nous retrouvons au carrefour du droit et de l'histoire, dans une démarche qui ne va pas sans susciter des problèmes qui sont tout à la fois philologiques et philosophiques. En effet, sous la couverture austère d'une histoire érudite d'un texte constitutionnel, Terminer la Révolution a une portée spéculative notable par sa méthode comme par les conséquences qui en découlent. Quelle est cette méthode?

Contribution à une «Histoire des Constitutions de France», collection sous la responsabilité de Robert Badinter et Didier Maus, le travail de Troper introduit subtilement un désaccord avec les termes qui sont supposés orienter cette recherche commune. Pour présenter l'esprit de la collection, quelques lignes en quatrième de couverture énoncent l'idée suivante : puisqu'une Constitution exprime «toujours une certaine conception du pouvoir d'État, et traduit un rapport de forces politiques », il y a intérêt à ce qu'on la regarde «comme un révélateur des idées et des forces politiques » qui l'ont rendue possible. Or - c'est là la singularité de ce travail - l'auteur annonce dès l'introduction qu'il ne veut pas lire la Constitution «comme la simple traduction des idéologies, des intérêts et des rapports de force» tels qu'on croit qu'ils ont pris forme à un moment donné de l'histoire. Il veut prendre en compte «la logique propre au travail constituant» (p. 14). Qu'est-ce à dire? Que l'élaboration d'un texte fondamental se présente comme une activité, douée de règles qui lui sont propres. Le rôle du chercheur sera alors de prendre au sérieux cette double dimension de l'élaboration constituante comme activité réglée, plutôt que de commencer par projeter sur le texte constitutionnel une lumière extérieure, venue de ce que l'on croit être l'idéologie régnante lors de sa rédaction, les intérêts des différentes parties ou encore les rapports de force entre elles. En bref, Troper souhaite présenter les textes préparatoires de la 
Constitution et la Constitution elle-même comme des faits simultanément réels et premiers, dignes non seulement d'être placés au centre de l'analyse, mais aussi d'être pris comme son principe.

Ce décalage infime par rapport à ce que l'on croirait être l'esprit de la collection est plein de conséquences. Notamment, il nous fait regarder sous un jour nouveau la place importante qu'occupent les annexes du livre : plus de 400 pages d'extraits des débats à la Convention nationale. Ces pages rendent accessibles au lecteur des documents sur lesquels s'appuie l'auteur, dans un ouvrage qui est une analyse historique des enjeux et, surtout, des discussions qui ont été à la base de la Constitution de l'an III. Le plus important, toutefois, est de reconnaître que commencer par les textes, et les prendre au sérieux, implique d'être très sensible aux changements constants auxquels ils sont soumis. Une fois admis ce principe, il apparaît que le texte constitutionnel définitivement consacré est différent aussi bien des projets qui ont contribué à sa préparation que des Constitutions qui l'ont précédé, et cela de manière parfois aussi imperceptible que définitive. C'est ce qui conduit Troper à ne pas faire l'histoire des idéologies et des intentions affichées par certains conventionnels, dont le discours de Baudin est un exemple, mais plutôt à s'intéresser à l'histoire de quelques maîtres mots dans lesquels, précisément, se joue quelque chose d'un autre ordre, à la fois technique et linguistique. Au centre de ce travail philologique, qui implique une véritable histoire des concepts, se trouvent des termes devenus aussi importants que souveraineté, gouvernement, séparation des pouvoirs ou encore citoyen, l'aventure intellectuelle que l'auteur nous propose étant de décortiquer les «étonnants glissements sémantiques » qu'ils subissent pendant les semaines qu'ont duré les débats préparatoires de la nouvelle loi.

Pour éclaircir le fonctionnement concret et les conséquences de la méthode de Troper par un exemple extrait du livre, prenons le terme auquel il consacre son chapitre $\mathrm{V}$, significativement intitulé «La mutation du concept de citoyen». Voici le cadre dont il part :

Dans la langue juridique contemporaine, le mot "citoyen" a deux sens : il désigne d'une part celui qui possède des droits politiques, notamment le droit de vote, d'autre part celui qui possède un certain nombre de droits civils liés au rattachement à l'État. Dans ce deuxième sens, citoyen s'op- 
pose à "étranger" et devient synonyme de national ou sujet d'État. Les citoyens ou nationaux ont des droits civils différents de ceux des étrangers. Il y a donc deux concepts distincts car si tout citoyen est nécessairement un national, tout national ne jouit pas des droits politiques et n'est donc pas citoyen dans le premier sens.

Mais, il n'en a pas toujours été ainsi. En réalité, on peut dater cette distinction de la Constitution de l'an III et du débat sur le suffrage. (p. 147)

Ayant décrit dans ces termes le cadre de sa recherche, l'auteur doit ensuite prouver que le concept de national naît en réalité à partir du débat à propos d'un autre concept, plus classique, celui de citoyen. Nous sommes sur le point d'assister à ce que l'auteur désigne comme «la mutation du concept». Celle-ci se déroule en trois moments. Premièrement, la Constitution de 1791 avait été construite sur la base de deux concepts de citoyen. D'abord étaient citoyens tous ceux qui, Français ou étrangers, hommes, femmes ou enfants, habitaient sur le territoire national, dans la mesure où ils jouissaient tous également des droits civils. Ensuite, dans un sens plus étroit, les citoyens étaient ceux qui possédaient, outre des droits civils, des droits politiques, notamment le droit de vote. De ce fait même, entre les Français il fallait introduire une distinction : certains pouvaient voter, d'autres non. La Constitution de 1791 consacre alors les premiers comme des citoyens actifs et les seconds comme des citoyens passifs, en même temps qu'elle détermine les conditions d'accès au vote sous la forme de paiement d'une contribution supérieure à un certain montant.

Deux ans plus tard, la Constitution montagnarde de 1793 «n'opposait plus entre actifs et passifs et tous étaient citoyens», rappelle Troper, qui ajoute aussitôt : "mais on distinguait parmi eux ceux qui étaient admis à exercer les droits politiques et ceux qui n'étaient pas admis à les exercer» (p. 147). En effet, il existe alors des citoyens qui «sont dans un état de soumission naturelle ou incapables d'autonomie» (p. 156); ce sont les femmes et les mineurs. Bien que tous soient pleinement citoyens au sens politique du mot, vu que «les droits politiques sont considérés comme naturels» (p. 156), certains citoyens ne peuvent pas exercer ces droits. En même temps, la distinction entre actifs et passifs ne peut plus servir à rendre compte de cette différence (et c'est pourquoi elle perd son sens), car il ne s'agit plus de séparer ceux qui paient une contribution de ceux qui n'en paient aucune. Si 
bien que la Constitution montagnarde conserve essentiellement les mêmes sens du mot citoyen et innove avant tout par l'instauration du suffrage universel. Si elle change la dénomination de la citoyenneté, parce qu'elle abandonne la distinction entre actifs et passifs, en réalité cela est «seulement une technique rédactionnelle, qui a une signification rhétorique, mais qui ne modifie pas le concept de citoyen. Tous sont citoyens, bien que tous ne soient pas admis à exercer les droits politiques» (p. 156). De ce point de vue, la métamorphose, conclut Troper, n'est pas substantielle.

En contrepartie, la Constitution de 1795 «exige à nouveau le paiement d'une contribution pour pouvoir voter et c'est cette exigence qui conduira à une mutation conceptuelle d'une portée considérable» (p. 147). Elle introduit deux nouveautés de taille, la notion de national et le droit de nationalité. L'auteur rend compte de ce fait à la fois historique, juridique et politique comme étant avant tout un fait discursif lié à des contraintes de logique interne aux débats. Dès que la Constitution prévoit le paiement d'une contribution directe comme l'une des conditions pour la citoyenneté, alors le corps du peuple est scindé. Désormais, «pour la première fois depuis le début de la Révolution, seuls ceux qui votent sont citoyens» (p. 148), et seuls ceux qui payent peuvent voter. Thomas Paine soulève à l'époque ce que Troper considère comme «une difficulté juridique et conceptuelle considérable» (ibid.) : si seuls ceux qui payent et qui votent sont citoyens, quel nom aura le reste du peuple? Et Paine ajoute : «Je veux parler de cette portion sur laquelle retombent tous les travaux, et sur laquelle tombera, par la suite, la charge des taxes indirectes» (cité par Troper, ibid.). C'est précisément pour cette autre partie du peuple qu'il faudra trouver un nom : «Si tous ceux qui ne peuvent pas voter ne sont pas citoyens, [...] ils sont tout simplement français, [...] ils sont nationaux.» (p. 159-160). Mais puisque «le terme de national sans doute n'existe pas [à l'époque] et l'on emploie, pour désigner ce concept, le seul terme dont on dispose, celui de citoyen», ce dernier "prend à ce moment sa double signification moderne de titulaire de droits politiques et de national» (p. 160).

Cette histoire, que nous ne pouvons ici que résumer de manière quelque peu formelle, illustre bien la méthode interprétative de l'auteur. Celle-ci consiste à mettre en avant les difficultés constantes, souvent techniques et menues, que les législateurs ont à résoudre, et dont la 
résolution porte avec elle de nouveaux problèmes dans un mouvement sans fin. Ce mouvement, toutefois, n'est pas indéterminé et il prend bien forme dans les textes constitutionnels qui se suivent et, on le voit maintenant, se distinguent parfois les uns des autres d'une manière on ne peut plus éthérée, quand les mots employés sont les mêmes (en l'occurrence, citoyen), mais qu'ils recouvrent des réalités divergentes.

Cette manière de procéder, mettant entre parenthèses les idéologies, les intérêts et les rapports de force, fait ressortir les contraintes argumentatives ou linguistiques dans lesquelles sont pris les constituants et dont découle rien de moins que la fabrication des principes constitutionnels. Le terme de fabrication peut paraitre problématique, mais ce choix terminologique se soutient solidement. L'idée de fabrication renvoie directement à la machinerie juridique, qui est une technique à résoudre des problèmes. Peut-être paraîtra-t-il étonnant que l'on puisse faire encore de nos jours l'histoire d'une Constitution sans avoir recours à ce qui est extérieur au texte constitutionnel, un cadre idéologique ou alors une couche de conditions de possibilité que nous pourrions être tentés d'appeler infrastructurelles (les intérêts de groupe et les rapports de force entre eux) et que ce texte est censé reproduire. Cependant, la prise en compte des textes comme s'ils étaient une sorte de monade, douée d'une histoire qui n'est ni celle des protagonistes qui les ont rédigés, ni celle des conditions qui les entourent, mais qui est celle des débats qui les ont préparés et des contraintes de rédaction qui leur ont donné forme, nous fait voir ce que l'on avait presque oublié, à force de rechercher les structures globales ou enveloppantes qui expliqueraient l'histoire des Constitutions : l'activité législative comme technique douée de règles et d'un mouvement qui lui sont propres. Tout se passe ici comme si la fabrication s'inscrivait en faux contre la détermination : les principes constitutionnels ne sont pas avant tout déterminés de l'extérieur par une conjoncture et encore moins par une structure idéologico-politique; ils sont avant tout le produit de contraintes discursives internes qui évoluent de façon immanente entre des moments différents et donc entre des Constitutions distinctes.

Certes la force de ce choix peut sembler être aussi sa faiblesse, et le lecteur se demander s'il ne serait pas quelque peu réducteur. Comment nier en effet l'influence des positions et des problèmes politiques, sociaux, économiques, moraux, sur l'activité constituante, 
comme d'ailleurs sur n'importe quel autre type d'activité? La question pourtant n'est pas là. Troper ne sait que trop bien que ces éléments se trouvent tous en jeu dans la vie d'un texte constituant. Aussi reconnaît-il qu' «il est incontestable que de nouvelles conceptions politiques se traduisent nécessairement par des règles nouvelles et que, en l'espèce, la Constitution de l'an III, réactionnaire en effet, a abandonné par exemple le droit de résistance ou le droit de secours» (p. 16). Seulement ces conceptions ne fournissent pas un point de départ consistant et encore moins une base solide pour l'analyse. L'auteur le souligne dès le début, lorsqu'il nous met en garde conte le «risque de circularité» auquel s'expose une histoire idéologique. «Il est en premier lieu très facile d'interpréter l'état d'esprit des conventionnels à la lumière des textes constitutionnels, puis les textes constitutionnels à la lumière de ce qu'on peut supposer l'état d'esprit de leurs auteurs » (p. 14). D'autant que, «deuxième risque de circularité», «on interprète les pratiques politiques sous le Directoire à la lumière d'une théorie que l'on impute aux conventionnels et que l'on affirme inscrite dans la Constitution, pour ensuite prouver la réalité de cette inscription dans la Constitution par les pratiques constitutionnelles, qui sont réputées en résulter» (ibid.). Éviter ces écueils est la tâche dont doit s'acquitter l'historien qui doute que les croyances aboutissent par elles seules à des règles. Pour lui, la théorie que produisent les conventionnels et que par la suite ils inscrivent dans la loi est tout simplement celle qui découle des débats, et non celle qui aurait précédé et orienté ces derniers, fût-elle celle de Rousseau. Ce n'est qu'à la fin que ce qui est inscrit dans la loi nous apparaît comme des théories, auxquelles souvent on associe les noms de certains conventionnels. La pratique juridique précède la théorie, et c'est seulement quand on a reconnu ce principe que l'on peut ensuite se tourner vers les théories, les idéologies, les intérêts et les rapports de force afin de les prendre comme des éléments d'une histoire.

Voilà, présenté à grands traits, ce qui condense l'originalité du livre de Troper à l'intérieur de son champ d'études, celui de l'histoire du droit et, spécifiquement, de l'histoire des Constitutions de la France. Sans doute la méthode mise en ouvre n'est-elle pas entièrement nouvelle en elle-même, et l'on pourrait affirmer qu'elle appartient encore à la bonne tradition du tournant linguistique. Pourtant, et à en juger par le tableau que donne l'auteur de l'état de la recherche sur la 
matière, elle est suffisamment rare dans cette sorte d'études pour susciter la controverse. Nous avons accordé un peu d'attention à l'examen des transformations du concept de citoyen qui, en 1795, ont mené au concept (mais pas au terme) de national. Dans son livre, la même technique est mise en œuvre pour étudier d'autres glissements sémantiques. Dans cet examen, l'auteur prend en compte le croisement de regards de l'époque entre l'Europe et l'Amérique, là où d'autres discussions constitutionnelles allaient marquer également les siècles à venir, jusqu'à nous. Pour toutes ces raisons, Terminer la Révolution est un ouvrage important, aussi bien par ses objets conceptuels que par la cohérence des principes qui l'orientent. Mais c'est aussi un livre provocant, par les effets qu'il souhaite avoir dans son champ d'études. C'est pourquoi il faut espérer qu'il ne passera pas inaperçu. 